\title{
OPERATIONS IN POLYADIC ALGEBRAS
}

\author{
BY \\ AUBERT DAIGNEAULT
}

\begin{abstract}
A new treatment of P. R. Halmos' theory of terms and operations in (locally finite) polyadic algebras (of infinite degree) is given that is considerably simpler than the original one.
\end{abstract}

Introduction. By a "polyadic algebra" we shall mean a "locally finite polyadic algebra of infinite degree". The theory of terms and operations in polyadic algebras has been developed by Halmos in [4] and [5] (see also [6]). The difficulty of [4] has proved to be a stumbling block for many a student of algebraic logic. The purpose of this paper is to present a considerably simplified treatment of the main results of [4] and [5] concerning terms and operations. There are three reasons that this treatment is easier and shorter than the original one of Halmos. First we use a simpler definition of term due to Léon LeBlanc [7, pp. 19-20]. Second we take advantage of the canonical method for extending a polyadic algebra to one with equality described by Halmos in $[5, \S 7]$. Finally full use is made of the representation theorem for polyadic algebras. For the purpose of substituting terms in operations, a representation theorem for the transformation system of terms is proved that links up with the notion of generalized ultrapower (equivalent to that of limit ultrapower) introduced in [1, p. 115].

1. Preliminaries. We shall use the notations of Halmos [3]-[5]. The theory of polyadic algebras with equality is assumed to be known up to and including the representation theorem. Let $F\left(X^{I}, B\right)$, where $B$ is a complete Boolean algebra, $X$ is a nonempty set and $I$ is the fixed infinite set of variables, denote the full functional (polyadic) algebra with value algebra $B$ and domain $X$. This is the set of all functions $p: X^{I} \rightarrow B$ with finite support under the Boolean pointwise operations and the quantifiers $(\exists J)$ and transformation endomorphisms $S(\alpha)$ defined by the equations

$$
\begin{gathered}
{[(\exists J) p](x)=\bigvee\left\{p(y) \mid y J_{*} x\right\}} \\
{[S(\alpha) p](x)=p(x \alpha),}
\end{gathered}
$$

where $J \subset I ; \alpha \in I^{I} ; x, y \in X^{I}$ and $y J_{*} x$ means $y_{j}=x_{j}$ for all $j \notin J$. The algebra

Received by the editors July 29, 1969 and, in revised form, August 10, 1970.

AMS 1969 subject classifications. Primary 0248; Secondary 0250.

Key words and phrases. Term, operation, constant, predicate polyadic algebra, Boolean algebra, transformation system, equality.

Copyright (C) 1971, American Mathematical Society 
$F\left(X^{I}, B\right)$ has an equality $E_{0}$ known as the functional equality and defined by the conditions

$$
\begin{aligned}
E_{0}(i, j)(x)=1 & \text { if } x_{i}=x_{j} \\
=0 & \text { if } x_{i} \neq x_{j}
\end{aligned}
$$

These conditions imply that

$$
\text { if } E_{0}(i, j)(x)=1 \text { then } x_{i}=x_{j} \text {. }
$$

A subalgebra of $F\left(X^{I}, B\right)$ may have an equality $E$ other than $E_{0}$. If $E$ has property (4) it is said to be reduced. The representation theorem for polyadic algebras proved in $[3,16.9$ and 17.1$]$ and $[5,6.7]$ can be stated thus:

THEOREM 1.1. Every polyadic algebra A with equality is isomorphic to a subalgebra $A^{\prime}$ of some $F\left(X^{I}, B\right)$ with a reduced equality and which is regular, i.e. such that every supremum (1) with $p \in A^{\prime}$ is attained for some $y$.

The case of polyadic algebras generally is reduced to that of polyadic algebras with equality by means of the following theorem proved in [5, \$7]:

THEOREM 1.2. For every polyadic algebra A there is a canonically defined polyadic algebra with equality $\bar{A}$ to which every constant or term of $A$ extends canonically.

The fact that every constant or term of $A$ extends to $\bar{A}$ is obvious from the construction of $\bar{A}$. This is so both for Halmos' original definitions of constant and of term (see [6, pp. 172, 178]) and for LeBlanc's definitions ([2, p. 89] and [7, p. 19]) which we shall adopt and which we now give.

Let $A$ be a polyadic algebra. A constant of $A$ is a function $c$ which assigns to each subset $K$ of $I$ a Boolean endomorphism $c(K)$ of $A$ (also denoted $S(c / K)$ ) in such a way that

(C1) $c(\phi)=1_{A}$, the identity map on $A$,

(C2) $c\left(K_{1} \cup K_{2}\right)=c\left(K_{1}\right) c\left(K_{2}\right)$,

(C3) $S(\alpha) c(K)=c(K)$ if $\alpha$ is identity on $I-K$,

(C4) $c(K) S(\sigma)=S(\sigma) c\left(\sigma^{-1} K\right)$,

for all $\sigma \in I^{I}$ and $K_{1}, K_{2} \subset I$.

A term of $A$ is a function $t$ which assigns to each subset $K$ of $I$ a Boolean endomorphism $t(K)$ (also noted $S(t / K)$ ) such that for some finite subset $J$ of $I$ (which we shall call a support of $t$ )

(T1) the restriction of $t$ to the subsets of $I-J$ is a $J$-constant of $A$, i.e. a constant of the algebra $A_{J}$ obtained from $A$ by fixing the variables in $J$; and

(T2) $t(K)=t(\sigma K) S(\sigma)$ for all transformations $\sigma$ such that $\sigma K \subset K$ and $\sigma$ is identity on $I-K$.

We shall call a term with support $J$ a $J$-term. Every variable $i$ induces an $\{i\}$-term which we will identify with $i$. One advantage of these conditions is that quantifiers do not appear in them so that they make sense in transformation algebras generally, 
and also that they are strictly equations between endomorphisms of $A$. LeBlanc's concept of constant was shown in [2, p. 97] to be equivalent to that of Halmos. That the same holds for terms will appear presently (see Corollary 2.6 below).

We insert here a simplified proof (proposed by the referee) that LeBlanc's concept of constant is equivalent to that of Halmos. The problem easily reduces to showing that $\exists(j) c(i)=c(i) \exists(j)$ for $i \neq j$. We may assume that our algebra has an equality. By the proof of $[3,10.1]$ we have

$$
\begin{aligned}
& \exists(j) c(i) p=\bigvee\{S(k / j) c(i) p: k \neq i\} \\
&=\bigvee\{c(i) S(k / j) p: k \neq i\} \quad \text { by }(\mathrm{C} 4), \\
& c(i) \exists(j) p=c(i) \vee\{S(k / j) p: k \neq i\} .
\end{aligned}
$$

From (1) and (2) we easily infer

$$
\exists(j) c(i) p \leqq c(i) \exists(j) p .
$$

Also, from (1) and (2) we see that it suffices to show that $c(i)$ is completely additive, i.e., that, if $\left\langle p_{t}: t \in T\right\rangle$ is a family of elements for which $\bigvee\left\{p_{t}: t \in T\right\}=r$ exists, then $\bigvee\left\{c(i) p_{t}: t \in T\right\}$ exists and equals $c(i) r$. (That this is so had also been proved by LeBlanc.) The following facts are useful first. The first two are easily established.

(4) If $i, j, k$ are distinct, then $c(j) E(i, j)=c(k) E(i, k)$,

(5) $c(i) c(j) E(i, j)=1$,

(6) if $i \neq j$ and $c(i) p \wedge q=0$, then $p \wedge \exists(i) q \wedge c(j) E(i, j)=0$.

To prove (6), first choose $k$ so that $k \neq i, j$ and $\exists(k) p=p$. Then

$$
\begin{aligned}
0=c(i) p \wedge q & =c(k) S(k / i) p \wedge q=c(k) \exists(i)(p \wedge E(i, k)) \wedge q \\
& \geqq \exists(i) c(k)(p \wedge E(i, k)) \wedge q \text { by }(3) \\
& =\exists(i)(p \wedge c(k) E(i, k)) \wedge q \\
& =\exists(i)(p \wedge c(j) E(i, j)) \wedge q \text { by (4). }
\end{aligned}
$$

The desired conclusion is then immediate.

Now we show that $c(i)$ is completely additive. Obviously $c(i) r$ is an upper bound for $\left\{c(i) p_{t}: t \in T\right\}$. Suppose that $q$ is another upper bound for $\left\{c(i) p_{t}: t \in T\right\}$. Then, for any $t \in T, c(i) p_{t} \wedge q^{\prime}=0$, so by (6), with $j \neq i$ chosen arbitrarily,

$$
p_{t} \wedge \exists(i) q^{\prime} \wedge c(j) E(i, j)=0 \text { for all } t \in T .
$$

Hence $r \wedge \exists(i) q^{\prime} \wedge c(j) E(i, j)=0$. It follows that $r \leqq \forall(i) q \vee c(j) E(i, j)^{\prime}$. Applying $c(i)$ to both sides and using (5) we get $c(i) r \leqq \forall(i) q \leqq q$, as desired.

We shall assume known the theory of constants including their relationship to equality. We also assume known the few facts concerning predicates that can be stated prior to the definition of terms, including the substitution of constants in predicates. All our prerequisites can be found in [2, pp. 57-101]. For the remainder of this paper $A$ will denote a fixed abstract polyadic algebra with equality $E$. 
2. Functional terms. In this section we relate terms of regular subalgebras of $F\left(X^{I}, B\right)$ having reduced equalities, with ordinary operations on $X$.

THEOREM 2.1. Let $T$ be an $n$-ary operation on $X$ and $\left(i_{1}, \ldots, i_{n}\right)$ a sequence of variables (not necessarily distinct). If, for $K \subset I, p \in F\left(X^{I}, B\right)$ and $x \in X^{I}$, we set

(1) $[S(t / K) p](x)=p(y)$ where $y K_{*} x$ and $y_{k}=T\left(x_{i_{1}}, \ldots, x_{i_{n}}\right)$ for all $k \in K$,

then $t$ is a term of $F\left(X^{I}, B\right)$ with support $J=\left\{i_{1}, \ldots, i_{n}\right\}$.

The proof of this theorem is a straightforward calculation. We shall leave cut all such calculations. This term $t$ will be denoted by $\hat{T}\left(i_{1}, \ldots, i_{n}\right)$ with no meaning attached to $\hat{T}$ alone for the time being. A term of this sort is called a functional term.

For a converse we need to be able to substitute terms in predicates, and two lemmas.

THeOREM 2.2. Let $P$ be an n-place predicate of $A$ and $t_{1}, \ldots, t_{n}$ terms of $A$ with the same support $J$. Assume that $i_{1}, \ldots, i_{n}$ are distinct variables in $I-J$. Then

$$
P\left(t_{1}, \ldots, t_{n}\right)=t_{1}\left(i_{1}\right) \cdots t_{n}\left(i_{n}\right) P\left(i_{1}, \ldots, i_{n}\right)
$$

is independent of the choice of $i_{1}, \ldots, i_{n}$.

Proof. This equation concerns the algebra $A_{J}$ (obtained by fixing the variables in $J$ ) in which the $t_{i}$ are constants. The proof in the case where the $t_{i}$ are constants is carried out in [2, p. 96] assuming $n=2$ for simplicity. Q.E.D.

LEMMA 2.3. Let $A_{1}$ be a polyadic algebra and $A_{2}$ be a subalgebra with equality $E$. Let $c_{i}$ be a constant of $A_{i}$ for $i=1,2$. If $E\left(c_{1}, c_{2}\right)=1$, then $c_{1}(K) p=c_{2}(k) p$ for all $p \in A_{2}$ and $K \subset I$, i.e. $A_{2}$ is closed under all $c_{1}(K)$, and the constant of $A_{2}$ so determined by restrictions of $c_{1}$ is $c_{2}$.

The proof is the same as that of 8.1 in [5]. Note that here $E\left(c_{1}, c_{2}\right)=c_{1}(i) E\left(i, c_{2}\right)$ by definition whereas $c_{2}(i) E\left(c_{1}, i\right)$ is undefined.

LEMMA 2.4. Every J-constant extends in at most one way to a J-term.

Proof. We first prove a sublemma: If $t$ is a J-term, $K \subset I, p$ is an element of support $L$ and $k \notin L$, then $t(K) p=t(K \cup\{k\}) p$. If $k \in K$ this is obvious. Assume $k \notin K$. Choose $\sigma$ such that $\sigma k \in K$ and $\sigma i=i$ if $i \neq k$. Then $\sigma(K \cup\{k\})=K$ and $\sigma$ satisfies the conditions of (T2) with $K \cup\{k\}$ in place of $K$. We have, as $L$ supports $p$ and $\sigma i=i$ for all $i \in L, S(\sigma) p=p$, and the result follows by (T2).

For the proof of the lemma itself, suppose that the $J$-constant $t$ has been extended to a $J$-term still denoted by $t$. Let $K \subset I$ and $p$ be of finite support $L$. If $k \in K-(L \cup J)$ and $\sigma$ is defined by the conditions $\sigma(i)=i$ for $i \in I-K$ and $\sigma(K)$ $=\{k\}$, then, by $(\mathrm{T} 2), t(K) p=t(k) S(\sigma) p$. Therefore, if $K-(L \cup J)$ is nonempty, the term $t$ is determined by the $J$-constant $t$. But, by the sublemma, we may assume that $K-(L \cup J)$ is nonempty. Q.E.D. 
We will prove only later that every $J$-constant does extend to a $J$-term so as to minimize our efforts.

Note that it is clear from the proof that if $t$ is a $J$-constant of a subalgebra $A_{2}$ of an algebra $A_{1}$, then $A_{2}$ is closed under the extension of $t$ to a $J$-term of $A_{1}$ if this extension exists.

THEOREM 2.5. Every term $t$ with support $J=\left\{i_{1}, \ldots, i_{n}\right\}$ of a regular subalgebra $A_{1}$ of $F\left(X^{I}, B\right)$ with reduced equality $E_{1}$ is obtained by restriction from a term $\hat{T}\left(i_{1}, \ldots, i_{n}\right)$ of $F\left(X^{I}, B\right)$ for some $T: X^{n} \rightarrow X$. Moreover $T$ is uniquely determined if $J$ and the sequence $\left(i_{1}, \ldots, i_{n}\right)$ are specified.

Proof. If $i \notin J$ we have $(\exists i) E_{1}(i, t)=1[2,10.15]$. Therefore $(\forall J)(\exists i) E_{1}(i, t)=1$. Since $E_{1}(i, t) \wedge E_{1}(j, t) \leqq E_{1}(i, j)$, for $j \notin J,(\forall J)(\exists ! i) E_{1}(i, t)=1$. From this, the regularity of $A_{1}$ and the assumption that $E_{1}$ is reduced, it follows that, for any $\left(x_{1}, \ldots, x_{n}\right) \in X^{n}$, there exists a unique $x \in X$ with the property: for all $y \in X^{I}$ such that $y_{i_{1}}=x_{1}, \ldots, y_{i_{n}}=x_{n}$, we have $E_{1}(i, t)(y)=1$ iff $y_{i}=x$. Set $T\left(x_{1}, \ldots, x_{n}\right)=x$.

By definition we have

$$
E_{1}\left[t, \hat{T}\left(i_{1}, \ldots, i_{n}\right)\right]=S\left[\hat{T}\left(i_{1}, \ldots, i_{n}\right) / i\right] E_{1}(t, i) \quad \text { if } i \notin J .
$$

Hence, by virtue of a property of the $J$-constant $\hat{T}\left(i_{1}, \ldots, i_{n}\right)$ of the algebra $F\left(X^{I}, B\right)$ with equality $E_{0}[2,10.18]$,

$$
E_{1}\left[t, \hat{T}\left(i_{1}, \ldots, i_{n}\right)\right]=(\exists i)\left[E_{1}(i, t) \wedge E_{0}\left(i, \hat{T}\left(i_{1}, \ldots, i_{n}\right)\right)\right] .
$$

By the definition of $T$, the right member of (4) is 1 . It follows from Lemma 2.3 that the $J$-constant $\hat{T}\left(i_{1}, \ldots, i_{n}\right)$ induces the $J$-constant $t$ in $A_{1}$. From this, Lemma 2.4 and the second remark following the proof of Lemma 2.4, it follows that the term $\hat{T}\left(i_{1}, \ldots, i_{n}\right)$ induces the term $t$ in $A_{1}$.

Finally to show the unicity of $T$, observe that the $T$ that we have defined is the only one that makes the right member of (4) equal to 1 . And, if $T_{1}$ satisfies the theorem, then $E_{1}\left[t, \hat{T}_{1}\left(i_{1}, \ldots, i_{n}\right)\right]=1$ while (4) is valid for any $T$. Q.E.D.

COROLLARY 2.6. The J-terms of a polyadic algebra in the foregoing sense are the same as its J-terms in the sense of Halmos [4, §4].

Proof. It follows directly from Theorems 1.2, 1.1, 2.5 and the easily verifiable fact that the terms $\hat{T}\left(i_{1}, \ldots, i_{n}\right)$ satisfy Halmos' definition, that any $J$-term in the foregoing sense is one in the sense of Halmos. The converse follows from the same statements except that, in Theorems 1.2 and 2.5 and their proofs, " $J$-term" is understood in the sense of Halmos. Q.E.D.

3. The transformation system of terms. By a transformation system we understand a set $\mathscr{T}$ together with a map $I^{I} \times \mathscr{T} \rightarrow \mathscr{T}$ that sends $(\sigma, t)$ onto $\sigma t$ in such a way that $\delta t=t$ and $\sigma_{1}\left(\sigma_{2} t\right)=\left(\sigma_{1} \sigma_{2}\right) t$ where $\delta$ is the identity map of $I$ onto itself. A subset $J$ of $I$ supports an element $t$ of $\mathscr{T}$ if $\sigma_{1} t=\sigma_{2} t$ whenever $\sigma_{1}\left|J=\sigma_{2}\right| J$, i.e. $\sigma_{1}$ and $\sigma_{2}$ have the same effect on $J$. A polyadic algebra is in an obvious sense a 
transformation system and this concept of support coincides with that of Halmos [2, $8.10]$. The concept of $n$-ary predicate of $\mathscr{T}$ is the same as for polyadic algebras and the basic properties of predicates of polyadic algebras remain unchanged for locally finite transformation systems generally $[2,8.11$ and 8.12$]$. In this section we endow the set of all terms of the polyadic algebras $A$ with a transformation structure and we shall henceforth reserve the letter " $\mathscr{T}$ " to denote that transformation system.

Let $t$ be the $J$-term of a regular subalgebra $A_{1}$ of $F\left(X^{I}, B\right)$ with reduced equality. Then, in the sense of Theorem $2.5, t=\hat{T}\left(i_{1}, \ldots, i_{n}\right)$ where $J=\left\{i_{1}, \ldots, i_{n}\right\}$, for some unique $T: X^{n} \rightarrow X$. It is easy to compute that

$$
S\left[\hat{T}\left(\sigma i_{1}, \ldots, \sigma i_{n}\right) / K\right] q=S(\alpha) S(t / \rho(K \cap L)) S(\rho) q
$$

where $q \in A_{1}, L$ is a finite support of $q, \rho$ is one-one on $L, \rho L \cap J=\varnothing, \alpha|J=\sigma| J$ and $\alpha \rho i=i$ for all $i \in L$. This shows that $A_{1}$ is closed under the term $\hat{T}\left(\sigma i_{1}, \ldots, \sigma i_{n}\right)$. We define $\sigma t$ to be the $\sigma J$-term of $A_{1}$ induced by $\hat{T}\left(\sigma i_{1}, \ldots, \sigma i_{n}\right)$. The representation theorem (Theorem 1.1) makes it possible to transfer this definition of $\sigma t$ to the abstract algebra $A$. This definition of $\sigma t$, for $t$ a term of $A$, is independent of the representation chosen since the right member of (1) is computed in $A$. Since, in $A_{1}$, $\sigma t=\hat{T}\left(\sigma i_{1}, \ldots, \sigma i_{n}\right)$, it is obvious that $\mathscr{T}$ becomes a transformation system.

We define an $n$-ary operation of $A$ to be an $n$-ary predicate of $\mathscr{T}$. If the regular subalgebra $A_{1}$ of $F\left(X^{I}, B\right)$ is closed under $\hat{T}\left(i_{1}, \ldots, i_{n}\right)$ for all $i_{1}, \ldots, i_{n} \in I$, then $\hat{T}$ can be viewed as an operation of $A_{1}$. Moreover, Theorem 2.5 means that any operation $Q$ of $A_{1}$ is of the form $\hat{T}$ for a unique $T$. We set $T=Q^{*}$.

The set of all functions $u: X^{I} \rightarrow X$ forms a transformation system if we set $(\sigma u)(x)=u(x \sigma)$ for $x \in X^{I}$. The set of all such functions with finite support is a locally finite transformation system denoted by $F\left(X^{I}, X\right)$. Any operation $T: X^{n} \rightarrow X$ induces a predicate $\check{T}$ of $F\left(X^{I}, X\right)$ defined by

$$
\left[\check{T}\left(i_{1}, \ldots, i_{n}\right)\right](x)=T\left(x_{i_{1}}, \ldots, x_{i_{n}}\right)
$$

where $x \in X^{I}$. Any predicate $P$ of $F\left(X^{I}, X\right)$ is of the form $\check{T}$ for a uniquely defined $T$ given by

$$
T\left(a_{1}, \ldots, a_{n}\right)=\left[P\left(i_{1}, \ldots, i_{n}\right)\right](x)
$$

where $\left(a_{1}, \ldots, a_{n}\right) \in X^{n}, i_{1}, \ldots, i_{n}$ are distinct variables and $x \in X^{I}$ is such that $x_{i_{1}}=a_{1}, \ldots, x_{i_{n}}=a_{n}$.

Every variable $i$ induces an element $\check{1}_{X}(i)$ of $F\left(X^{I}, X\right), 1_{X}$ being the identity function on $X$.

THEOREM 3.1. Let $\mathscr{T}(X, I, B)$ be the transformation system of functional terms of $F\left(X^{I}, B\right)$. Then an isomorphism $\phi: \mathscr{T}(X, I, B) \rightarrow F\left(X^{I}, X\right)$ is defined by the equation $\phi\left[\hat{T}\left(i_{1}, \ldots, i_{n}\right)\right]=\check{T}\left(i_{1}, \ldots, i_{n}\right)$. We will identify these two systems by this isomorphism. 
Proof. The only not quite trivial part of the statement is that $\phi$ is well defined and injective. This means that for any two operations $T_{1}: X^{n} \rightarrow X$ and $T_{2}: X^{m} \rightarrow X$ and any two sequences of variables $\left(i_{1}, \ldots, i_{n}\right)$ and $\left(j_{1}, \ldots, j_{m}\right)$,

$$
\hat{T}_{1}\left(i_{1}, \ldots, i_{n}\right)=\hat{T}_{2}\left(j_{1}, \ldots, j_{m}\right)
$$

iff

$$
\check{T}_{1}\left(i_{1}, \ldots, i_{n}\right)=\check{T}_{2}\left(j_{1}, \ldots, j_{m}\right) .
$$

Note that both members of (1) are $\left\{i_{1}, \ldots, i_{n}, j_{1}, \ldots, j_{m}\right\}$-terms and that, by virtue of $[5,8.1]$ (see Lemma 2.3), (1) means that

$$
E_{0}\left[\hat{T}_{1}\left(i_{1}, \ldots, i_{n}\right), \hat{T}_{2}\left(j_{1}, \ldots, j_{m}\right)\right]=1 .
$$

Since $E_{0}$ is reduced, both (2) and (3) mean that, for all $x \in X^{I}$,

$$
T_{1}\left(x_{i_{1}}, \ldots, x_{i_{n}}\right)=T_{2}\left(x_{j_{1}}, \ldots, x_{j_{m}}\right)
$$

(of course, for (4) to happen, $T_{1}$ and $T_{2}$ must be very closely related: $T_{2}$ must be obtainable from $T_{1}$ by relocating the relevant places and by leaving out or adding some irrelevant places). Q.E.D.

The definition of the image of a term of a polyadic algebra $A_{1}$ by a polyadic onto homomorphism $A_{1} \rightarrow A_{2}$ poses no difficulty. This is done exactly as in the case of constants $[3,15.3]$.

A regular reduced representation of $A$ is a polyadic homomorphism of $A$ onto a regular subalgebra $A_{1}$ of some $F\left(X^{I}, B\right)$ with reduced equality $E_{1}$. Theorems 2.5 and 3.1 make it possible to define in an obvious way, for any such representation of $A$, a companion representation for $\mathscr{T}$ which will be a transformation homomorphism from $\mathscr{T}$ into $F\left(X^{I}, X\right)$.

This generalizes to the case where $E_{1}$ is not reduced: for that one must consider the system $F\left(X^{I}, Y\right)$ where $Y$ is the quotient of $X$ by the equivalence relation $\sim$ on $X$ determined by $E_{1}: a \sim b$ iff $\left[E_{1}(i, j)\right](x)=1$ for $x \in X^{I}$ such that $x_{i}=a$ and $y_{j}=b$ for two distinct variables $i$ and $j$.

The natural surjection $X \rightarrow Y$ induces a surjection $X^{I} \rightarrow Y^{I}$ which, by composition, yields a transformation monomorphism $F\left(Y^{I}, Y\right) \rightarrow F\left(X^{I}, Y\right)$ and a polyadic monomorphism $F\left(Y^{I}, B\right) \rightarrow F\left(X^{I}, B\right)$. The (regular) representation $A \rightarrow F\left(X^{I}, B\right)$ splits uniquely through a (regular) reduced representation $A \rightarrow F\left(Y^{I}, B\right)$ so that $A \rightarrow F\left(X^{I}, B\right)=A \rightarrow F\left(Y^{I}, B\right) \rightarrow F\left(X^{I}, B\right)$. The companion representation $\mathscr{T} \rightarrow F\left(Y^{I}, Y\right)$ of the reduced representation yields a compansion representation $\mathscr{T} \rightarrow F\left(X^{I}, Y\right)\left[=\mathscr{T} \rightarrow F\left(Y^{I}, Y\right) \rightarrow F\left(X^{I}, Y\right)\right]$ of the given representation.

The notion of the $K$-compression of an $I$-transformation system can be defined just as with polyadic algebras, $K$ being a subset of $I$. The $K$-compression $\mathscr{T}^{(K)}$ of $\mathscr{T}$ is the set of $J$-terms of $A$ such that $J \subset K$. A regular (not necessarily reduced) representation of $A$ into $F\left(X^{I}, B\right)$ will induce a companion representation of $\mathscr{T}^{(K)}$ into $F\left(X^{K}, Y\right)$. In [1, p. 115] generalized ultrapowers of a set $Y$ have been defined as 
quotients of sets of the form $F\left(X^{K}, Y\right)$ by (Boolean) ultrafilters of the ( $K$-polyadic) algebra of all functions with finite support from $X^{K}$ to $2=\{0,1\}$.

4. Terms in operations. If $Q$ is an $n$-ary operation of $A$ and $t_{1}, \ldots, t_{n} \in \mathscr{T}$, we wish to define the term $t=Q\left(t_{1}, \ldots, t_{n}\right)$. We wish that the definition be such that

$$
S(t / K) p=S\left(t_{1} / i_{1}\right) \cdots S\left(t_{n} / i_{n}\right) S\left[Q\left(i_{1}, \ldots, i_{n}\right) / K\right] p
$$

whenever $i_{1}, \ldots, i_{n}$ are distinct variables none of which is in a common support of $p, t_{1}, \ldots, t_{n}$ and $K$ is an arbitrary subset of $I$. To avoid the rather cumbersome problem of showing directly that (1) is an unambiguous definition of a term $t$ we rely on the representation theorem (Theorem 1.1) and on the representation theorem for the system of terms $\mathscr{T}(X, I, B)$ (Theorem 3.1).

Identify momentarily $A$ with a regular subalgebra of some $F\left(X^{I}, B\right)$ with reduced equality. Then $Q$ is essentially $\hat{T}$ for some $T: X^{n} \rightarrow X$. Before transforming $\hat{T}$ from a function $I^{n} \rightarrow \mathscr{T}$ into an $n$-ary operation on $\mathscr{T}$, we transorm $\check{T}$ from a function $I^{n} \rightarrow F\left(X^{I}, X\right)$ into an $n$-ary operation on $F\left(X^{I}, X\right)$. For this we let $u_{1}, \ldots, u_{n} \in F\left(X^{I}, X\right)$ and $x \in X^{I}$ and set

$$
\left[\check{T}\left(u_{1}, \ldots, u_{n}\right)\right](x)=T\left(u_{1}(x), \ldots, u_{n}(x)\right) .
$$

Now define $\hat{T}\left(t_{1}, \ldots, t_{n}\right)$ by

$$
\hat{T}\left(t_{1}, \ldots, t_{n}\right)=\phi^{-1}\left[\check{T}\left(\phi t_{1}, \ldots, \phi t_{n}\right)\right]
$$

where $\phi$ is the isomorphism from the system of functional terms of $F\left(X^{I}, B\right)$ onto the system $F\left(X^{I}, X\right)$. Note that, a priori, (3) defines a term $t$ of $F\left(X^{I}, B\right)$ under which $A$ might not be closed. That $A$ is closed under $t$ follows from (1) which can be verified for $p \in F\left(X^{I}, B\right)$ by an easy computation. For this, note that, if $J=\left\{i_{1}, \ldots, i_{m}\right\}$ is a common support of $t_{1}, \ldots, t_{m}$ the term $t$ is $\hat{U}\left(i_{1}, \ldots, i_{m}\right)$ where $U$ is an $m$-ary operation on $X$ defined by

$$
U\left(x_{1}, \ldots, x_{m}\right)=\left[\check{T}\left(\phi t_{1}, \ldots, \phi t_{n}\right)\right](y)
$$

where $y \in X^{I}$ is such that $x_{k}=y_{j_{k}}$ for $k=1, \ldots, m$ (this definition does not depend on $y$ ). That the definition of $Q\left(t_{1}, \ldots, t_{n}\right)$ does not depend on the representation chosen for $A$ also follows from (1).

5. Operations and monovalent predicates. An $(n+1)$-ary predicate $P$ of $A$ is monovalent in its first place if, for distinct variables $k, i, i_{1}, \ldots, i_{n}$,

$$
(\exists i) P\left(i, i_{1}, \ldots, i_{n}\right)=1 \text {. }
$$

and

$$
P\left(i, i_{1}, \ldots, i_{n}\right) \wedge P\left(k, i_{1}, \ldots, i_{n}\right) \rightarrow E(i, k)=1 .
$$

The conjunction of the left members of these two equations is written

$$
(\exists ! i) P\left(i, i_{1}, \ldots, i_{n}\right) \text {. }
$$


The following theorem has first been proved by Halmos $[5,9.2]$. The proof given here generalizes that given in $[2,11.5]$ for the case of constants.

THEOREM 5.1. The n-ary operations $T$ of $A$ and the $(n+1)$-ary predicates $P$ of $A$ monovalent in their first place are in one-one correspondence by the following relations:

$$
E\left[i, T\left(i_{1}, \ldots, i_{n}\right)\right]=P\left(i, i_{1}, \ldots, i_{n}\right)
$$

for arbitrary variables $i, i_{1}, \ldots, i_{n}$, and

$$
S\left[T\left(i_{1}, \ldots, i_{n}\right) / K\right] p=(\exists i)\left[S(i / K) p \wedge P\left(i, i_{1}, \ldots, i_{n}\right)\right]
$$

where $i \notin L \cup\left\{i_{1}, \ldots, i_{n}\right\}, L$ being a support of $p$.

Proof. Let $T$ be given and define $P$ by (3). We have (1) by [2, 10.15] and (2) by applying $S\left[T\left(i_{1}, \ldots, i_{n}\right) / j\right]$ to $E(i, j) \wedge E(k, j) \leqq E(i, k)$. We have (4) with $K \cup I-J$, where $J=\left\{i_{1}, \ldots, i_{n}\right\}$, by $[2,10.18]$. The case of an arbitrary $K$ follows from this special one: for let $k \notin L \cup\left\{i_{1}, \ldots, i_{n}\right\}$, then as in the proof of Lemma 2.4, with $t=T\left(i_{1}, \ldots, i_{n}\right)$,

$$
\begin{aligned}
S(t / K) p & =S(t / k) S(k / K) p \\
& =(\exists i)\left[S(i / k) S(k / K) p \wedge P\left(i, i_{1}, \ldots, i_{n}\right)\right] \\
& =(\exists i)\left[S(i / K) p \wedge P\left(i, i_{1}, \ldots, i_{n}\right)\right]
\end{aligned}
$$

Hence the map $T \mapsto P$ is injective.

Let now $P$ be an $(n+1)$-ary predicate monovalent in its first place. Assuming that (4) does define an operation $T$ and letting $K=\{i\}$ and $p=E(j, i)$ in (4), we have

$$
E\left[j, T\left(i_{1}, \ldots, i_{n}\right)\right]=(\exists i)\left[E(j, i) \wedge P\left(i, i_{1}, \ldots, i_{n}\right)\right]=P\left(j, i_{1}, \ldots, i_{n}\right) .
$$

Hence the map $T \mapsto P$ is onto.

The proof that (4) does define an operation $T$ begins with an easy modification of a part of the proof of 9.2 in [5]. For this purpose, identify $A$ with a regular subalgebra of some $F\left(X^{I}, B\right)$ with reduced equality. Then for all $x_{1}, \ldots, x_{n} \in X$ there is a unique $x \in X$ such that $\left[P\left(i, i_{1}, \ldots, i_{n}\right)\right](y)=1$ whenever $y_{i}=x$ and $y_{i_{k}}=x_{k}$ for $k=1, \ldots, n$. Define $Q: X^{n} \rightarrow X$ by setting $Q\left(x_{1}, \ldots, x_{n}\right)=x$. Then it is clear that

$$
P\left[\hat{Q}\left(i_{1}, \ldots, i_{n}\right), i_{1}, \ldots, i_{n}\right]=1 .
$$

Now apply $S\left[\hat{Q}\left(i_{1}, \ldots, i_{n}\right) / k\right]$ to

$$
P\left(i, i_{1}, \ldots, i_{n}\right) \wedge P\left(k, i_{1}, \ldots, i_{n}\right) \leqq E(i, k)
$$

to get, using (5),

$$
P\left(i, i_{1}, \ldots, i_{n}\right) \leqq E\left[i, \hat{Q}\left(i_{1}, \ldots, i_{n}\right)\right]
$$


On the other hand,

$$
P\left(k, i_{1}, \ldots, i_{n}\right) \wedge E(i, k)=P\left(i, i_{1}, \ldots, i_{n}\right) \wedge E(i, k) .
$$

And hence,

$$
P\left(k, i_{1}, \ldots, i_{n}\right) \wedge E(i, k) \leqq P\left(i, i_{1}, \ldots, i_{n}\right) .
$$

Applying $S\left[\hat{Q}\left(i_{1}, \ldots, i_{n}\right) / k\right]$ and using (5) again

$$
E\left[i, \hat{Q}\left(i_{1}, \ldots, i_{n}\right)\right] \leqq P\left(i, i_{1}, \ldots, i_{n}\right) .
$$

Hence

$$
E\left[i, \hat{Q}\left(i_{1}, \ldots, i_{n}\right)\right]=P\left(i, i_{1}, \ldots, i_{n}\right) .
$$

Now assume first that $A$ is simple. Then $B$ can be taken to be 2. (This, incidentally, follows from 1.1 since any regular representation $f$ of $A$ into $F\left(X^{I}, B\right)$ yields a regular representation $\bar{f}$ of $A$ into $F\left(X^{I}, B / N\right)$ for any ideal $N$ of $B$ simply by setting $(\bar{f} p)(x)=(f p)(x) / N$ and this representation $\bar{f}$ can be reduced.) Then $E$ is the functional equality and (4) holds with $\hat{Q}$ instead of $T$ in view of (6) as can easily be calculated. When $A$ is not simple (4), with $T=\hat{Q}$, is not so obvious though it is true. It holds if $K \subset I-\left\{i_{1}, \ldots, i_{n}\right\}$ by virtue of the properties of constants. If we knew that every $J$-constant extends to a $J$-term then we could easily claim the validity of (4) with $T=\hat{Q}$ by virtue of this, (T2) and the first paragraph of the proof of Lemma 2.4 .

The case where $A$ is not simple will follow by semisimplicity. For this we digress a little. By the direct product of a family of polyadic algebras we understand the subalgebra of all elements with finite support in the (possibly not locally finite) cartesian product algebra with the "operations" $(\exists J)$ and $S(\alpha)$ performed componentwise. This direct product, together with the obvious projection epimorphisms, is the product in the category of (equality) algebras with (equality) homomorphisms. A predicate $P$ of such a product $C=\prod\left\{C_{h} \mid h \in H\right\}$ can be identified with a family $\left\{P_{h} \mid h \in H\right\}$ of predicates of the $C_{k}$ by the equation

$$
P\left(i_{1}, \ldots, i_{n}\right)=\left\{P_{h}\left(i_{1}, \ldots, i_{n}\right)\right\} \text {. }
$$

Similarly, for operations we have the following obvious

LEMMA 5.2. If $C=\prod\left\{C_{h} \mid h \in H\right\}$ is a direct product of polyadic algebras and if, for all $h, T_{h}$ is an n-ary operation of $C_{h}$, then an $n$-ary operation $T$ of $C$ is defined by the equation

$$
S\left[T\left(i_{1}, \ldots, i_{n}\right) / K\right] p=\left\{S\left[T_{h}\left(i_{1}, \ldots, i_{n}\right) / K\right] p_{h}\right\}
$$

where $p=\left\{p_{h}\right\} \in C$.

From the semisimplicity of polyadic algebras $[3,9.3]$ it follows that any polyadic algebra is isomorphic to a subalgebra of a direct product of simple algebras in the usual manner. The fact that (4) defines an operation in general follows from the above remarks. This completes the proof of Theorem 5.1. 
An immediate consequence which could also have been proved earlier (see Lemma 2.4) with some independent labor is the following

COROLlARY 5.3. Every J-constant extends to a J-term.

Proof. Let $J=\left\{j_{i}, \ldots, j_{n}\right\}$ and $P$ be the unique predicate such that $P\left(i, j_{1}, \ldots, j_{n}\right)$ $=S(c / k) E(i, k)$ where $i, k \in I-J$. Then $P$ is monovalent in its first place and if $T$ is obtained by 5.1 then $T\left(j_{1}, \ldots, j_{n}\right)$ extends $c$. Q.E.D.

\section{REFERENCES}

1. A. Daigneault, On automorphisms of polyadic algebras, Trans. Amer. Math. Soc. 112 (1964), 84-130. MR 29 \#45.

2. - Théorie des modèles en logique mathématique, 2ième éd., Presses Univ. Montréal, Montréal, 1967. MR 40 \#31.

3. P. R. Halmos, Algebraic logic. II. Homogeneous locally finite polyadic Boolean algebras of infinite degree, Fund. Math. 43 (1956), 255-325. MR 19, 112.

4. - Algebraic logic. III. Predicates, terms, and operatives in polyadic algebras, Trans. Amer. Math. Soc. 83 (1956), 430-470. MR 19, 113.

5. - Algebraic logic. IV. Equality in polyadic algebras, Trans. Amer. Math. Soc. 86 (1957), 1-27. MR 19, 830.

6. ——, Algebraic logic, Chelsea, New York, 1962. MR 24 \#A1808. (This contains [3], [4] and [5].)

7. L. LeBlanc, Représentabilité et définissabilité dans les algèbres transformationnelles et dans les algèbres polyadiques, Presses Univ. Montréal, Montréal, 1966. MR 40 \#4090.

UNIVERSITÉ DE MONTRÉAL, Montréal, Qú́bec, Canada 